\title{
Vedic ALU using Area Optimised Urdhva Triyambakam Multiplier
}

\author{
Anshul Khare \\ PG student, Department of \\ Electronics \& Communication \\ IES College Bhopal, India
}

\author{
Ashish Raghuwanshi \\ Asst. professor, Department of \\ Electronics \& Communication \\ IES College Bhopal, India
}

\author{
Ruchi Gupta \\ Asst. professor, Department of \\ Electronics \& Communication \\ IES College Bhopal, India
}

\begin{abstract}
Multiplication has some limits and to overcome these limitations a new approach has been describe and designed a Vedic multiplier with proposed unique addition structure, which is used to perform addition of partially generated products. To meet main concern 'area' and 'speed' we have came up with a need particular high speed ALU, the speed of ALU greatly depends upon the speed of multiplication unit used in it. There are numerous multiplication techniques exist now a days at algorithmic and structural level. It is been shown that Vedic multiplication is the fastest multiplication method but there are some other multiplication techniques which are batter then vedic multiplication in terms of chip area. This Proposed work is a unique architecture of 16 bit vedic with combination of 4 bit vedic multiplications and that 4 bit multiplication is been have developed with a unique addition structure. The observed results are been very good and optimised. Later on ALU module is been developed. The tool used for the designing is Xilinx XST and the target platform for validation is Vertex family vertex-4 FPGA, the preferred language is VHDL.
\end{abstract}

\section{Keywords}

Vedic mathematics, Carry save adder, Arithmetic and logical unit, Urdhva Tiryambakam sutra, RTL, User constrain file

\section{INTRODUCTION}

Now days we are living in digital world, where all operations get performed with more reliably and highest accuracy by digital signal processor. The key element of all the processors like Microprocessor, Microcontroller, DSP processor etc is ALU. Every digital domain based technology depends upon the operations performed by ALU either partially or whole. Speed is the most prominent factor of processor and

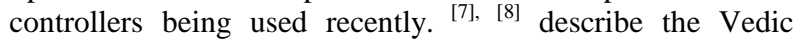
mathematics from beginning and discuss all operations.

By improving the ALU unit we can develop efficient the Digital Signal Processor, for that proposed Arithmetic unit appears very useful ${ }^{[6]}$. One of the major purposes of Vedic mathematics is to execute the difficult calculations in simple way, even manageable orally without much use of pen and paper.

\section{VEDIC MULTIPLICATION TECHNIQUE}

In conventional mathematics Vedic mathematics is used to reduce the complex calculation in the to very simple one. The human mind works on the natural principles on which the Vedic formulae are based. Vedic Mathematics is a method of arithmetic rules which allows much more efficient speed implementation. It also provides few effective algorithms which can be applied to many branches of engineering like computing.

\subsection{Urdhva Tiryambakam Sutra}

The proposed Vedic multiplier is based on the "Urdhva Tiryambakam" sutra. These formulas are mainly used for multiplication of two decimal numbers ${ }^{[6]}$. In present paper we apply this algorithm on binary numbers. This multiplication formula can be apply to all cases of multiplication .The term "Urdhva Triyambakam" originated from two Sanskrit words Urdhva and Triyambakam which means "Vertically" and "Crosswise" respectively ${ }^{[5]}$. This method is based on the concept in which all partial products are generated concurrently. This algorithm can be applicable for $\mathrm{n} \times \mathrm{n}$ bit number. All the partial products and their sums are parallely calculated; the multiplier does not dependents on the processors clock frequency. It can be easily layout in microprocessors due to its regular structure, and designers can easily identify these problems to avoid device failures. Processing power of multiplier can be easily enhanced by increasing width of the input and output data bus. It can be easily layout in silicon chip due to its regular structure. The multiplier based on "Urdhva Tiryambakam" sutra has the advantage that in comparison to other multipliers gate delay and area increases very slowly as the number of bits increases. So this multipier is space, time and power efficient.

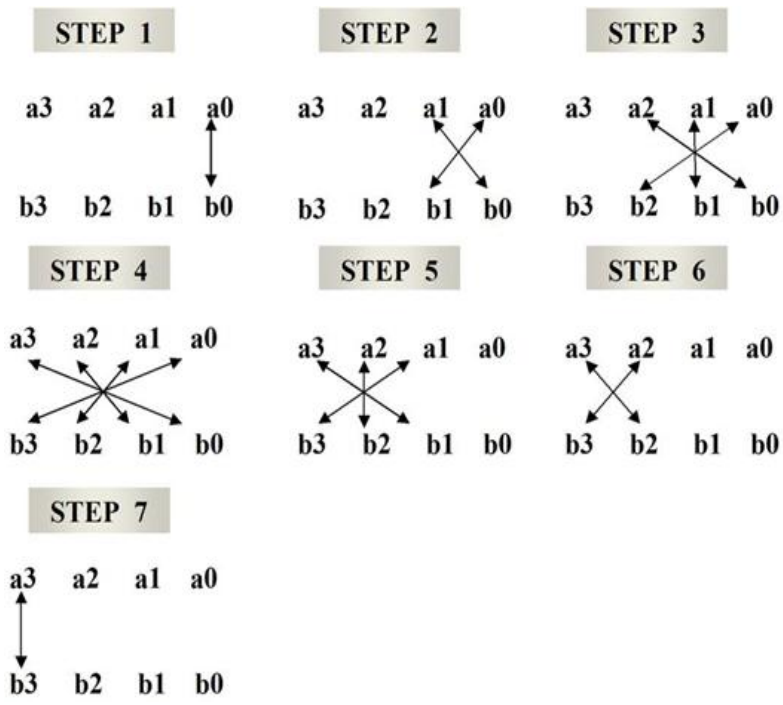

Figure 1: Multiplication of two 4 bit numbers using Urdhva Tiryambakam method 


\section{PROPOSED ARITHMETIC UNIT}

Proposed 16x16 bit Arithmetic Unit is given in the figure 2.

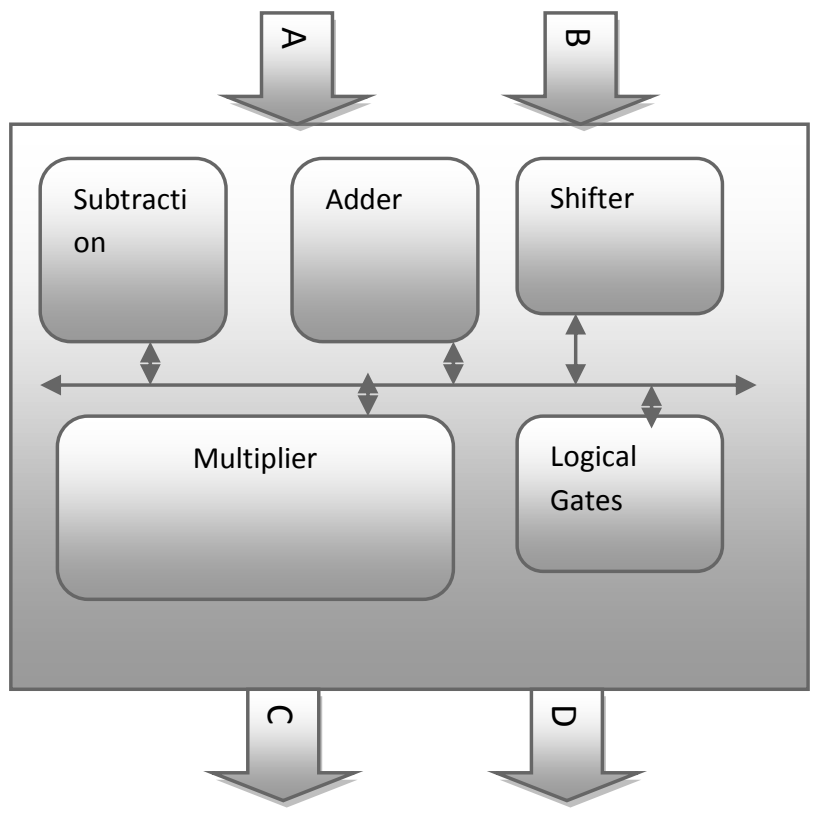

Figure 2: Arithmetic Logic Unit Module

Here the A and B are the two 16 bit inputs of proposed Arithmetic Unit. And other portion of the design includes Adder, Subtractor, Multiplier, and ALU. Product and Accumulated product are 32 bit output while differences, $\mathrm{S}$ are 16 bit output. Proposed work did not focus on the designing of the adder and subtractor circuits as these are not consider modules which consumes large amount of area and power in ALU. But after detailed study it is been found that normally, carry ripple adders can be used when it required to meet timing constraints because they are easy to build and compact.

\section{RESULTS}

Till now we have successfully new design for 4 bit Vedic multiplier with the help of new addition structure and also design $8 \times 8$ and $16 \times 16$ with help of $4 \times 4$ multiplication. Proposed work is a design of ALU with the help of modified new urdhava triyambakam Vedic multiplication method (i. e. proposed multipliers). After successful implementation $4 \times 4$, $8 \times 8$ and $16 \times 16$ an ALU is been design and Figure 3 below shows the simulation results for the ALU design modules and it shows the results for multiplication, addition and subtraction.
Table 1: results observed for design

\begin{tabular}{|l|l|}
\hline \multicolumn{2}{|l|}{ Family : Vertex 4 FPGA Family } \\
\hline Observed results for proposed vedic 4x4 \\
\hline No of Slices & 18 \\
\hline No of 4 input LUT & 31 \\
\hline No of bounded IOBs & 16 \\
\hline Logical Delay & $4.891 \mathrm{~ns}$ \\
\hline Observed results for proposed vedic 8x8 \\
\hline No of Slices & 90 \\
\hline No of 4 input LUT & 158 \\
\hline No of bounded IOBs & 32 \\
\hline Logical Delay & $6.448 \mathrm{~ns}$ \\
\hline Observed results for proposed vedic ALU 16x16 \\
\hline No of Slices & 452 \\
\hline No of 4 input LUT & 803 \\
\hline No of bounded IOBs & 68 \\
\hline Logical Delay & 9.007 ns \\
\hline
\end{tabular}

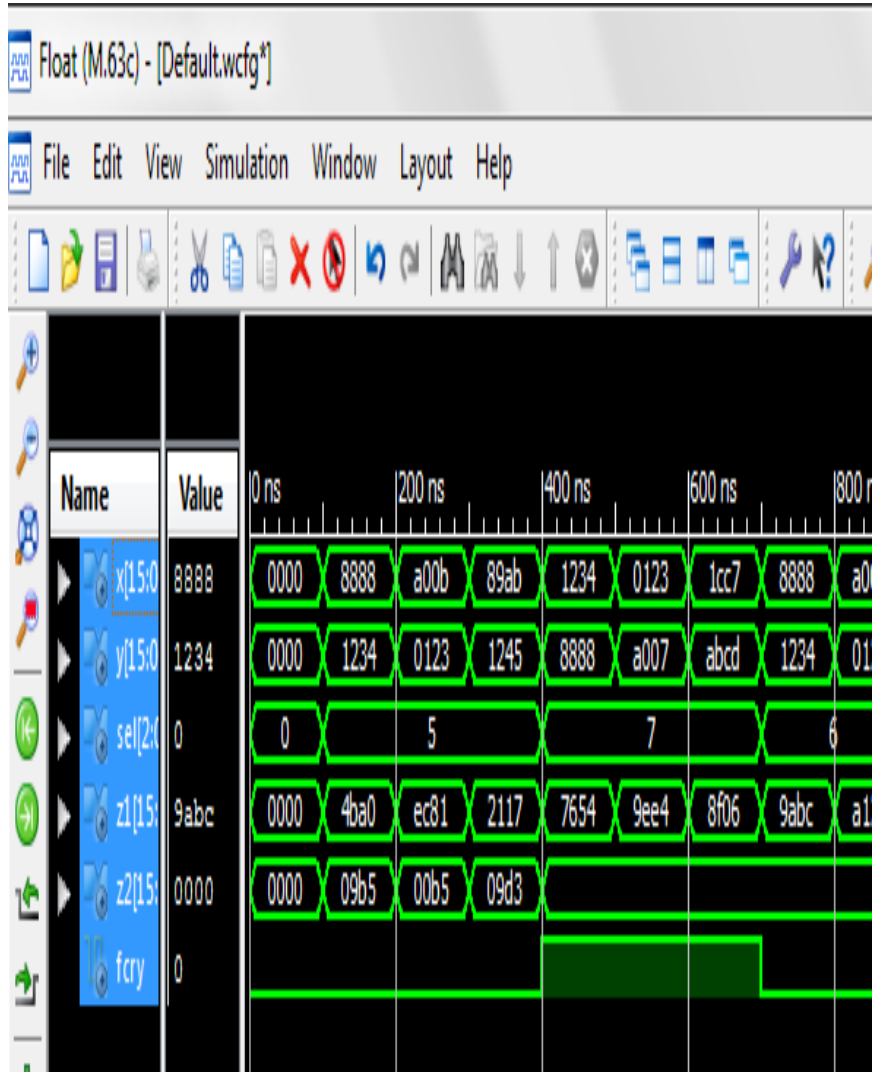

Figure 3: simulation results of ALU

The results are been produced after RTL entry in Xilinx EDA tool. Simulation is done on Xilinx ISE and results are been verified correctly. 
Table 2: full comparative results

\begin{tabular}{|c|c|c|c|}
\hline \multicolumn{4}{|c|}{ Platform used is vertex family FPGA } \\
\hline Design & Logical Delay & Slice & LUT \\
\hline $\begin{array}{c}\text { Proposed Vedic } 4 \\
\text { bit }\end{array}$ & $4.891 \mathrm{~ns}$ & 18 & 31 \\
\hline Base 1 (4x4) & 8.387 & 19 & - \\
\hline Base 2(4x4) & - & - & - \\
\hline $\begin{array}{c}\text { Proposed Vedic } 8 \\
\text { bit }\end{array}$ & $6.448 \mathrm{~ns}$ & 90 & 158 \\
\hline $\begin{array}{c}\text { Base 1(8x8) } \\
\text { Base 2(8x8) }\end{array}$ & $11.886 \mathrm{~ns}$ & - & - \\
\hline Proposed 16 bit & $9.007 \mathrm{~ns}$ & 452 & - \\
\hline Base (16x16) & $15.718 \mathrm{~ns}$ & - & - \\
\hline Base 2 (16x16) & $23.064 \mathrm{~ns}$ & - & - \\
\hline & $\begin{array}{c}\text { Combinational } \\
\text { path delay }\end{array}$ & & \\
\hline $\begin{array}{c}\text { Proposed Vedic 8 } \\
\text { bit }\end{array}$ & $13.753 \mathrm{~ns}$ & 90 & 158 \\
\hline Base 3(8x8) & 13.753 & - & - \\
\hline
\end{tabular}

Above it can observe that proposed work has batter results in aspect of area (i.e. less number of Slices) and speed (i.e. logical delay) in 4 bit multiplication as compare to base 3 paper.

Above one can easily observe that proposed work is batter in aspect of speed (i.e. logical delay) in 8 bit as compare to base 1 , base $2 \&$ base 3 papers.

Proposed is a design of 4 bit Vedic multiplier (our actual research work) and use it to design 16 bit ALU. So one should make comparison with 4 bit Vedic only, rest of comparison are also been made.

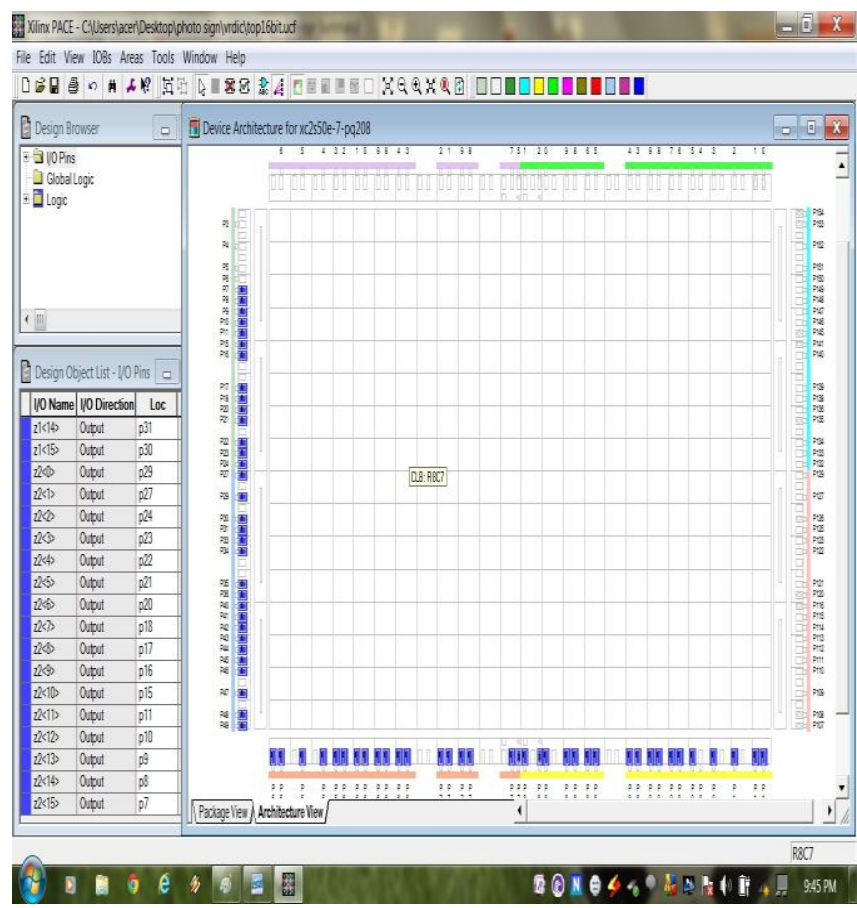

Figure 4: FPGA floor plan of proposed work
Figure 4 above is the floor plan of proposed work which is designed for generation UCF (user constrain file) for final validation of design on FPGA.

\section{CONCLUSION}

In this paper we proposed a novel architecture for the $16 \times 16$ bit Multiplier and a 16 bit ALU which provides somewhat better results as compare to the available Vedic multiplier or all other Multiplier. Proposed design can also be used for optimizing the multiply and accumulate unit of DSP. And so the optimum designs can be used for FFT, DFT, IIR, and FIR whose performance is dependent on the speed of ALU unit. There are so many applications where speed is more concerns than any other things like signal processing in satellite GPS based systems disaster management system etc. Proposed work give a solution for highly speed ALU and as know ALU is the key element part of software based embedded or general (i.e. computer) systems. The Future scope of this work can be extending for 32 bit, 64 bit etc.

\section{ACKNOWLEDGMENTS}

The authors would like to thank faculties of ECE Department of IES College of Technology, Bhopal for their kind guidance and support.

\section{REFERENCES}

[1] Anthony O'Brien and Richard Conway, "Lifting Scheme Discrete Wavelet Transform Using Vertical and Crosswise Multipliers", ISSC, June 18-19, 2008 , Galway,

[2] A.P. Nicholas, K.R Williams, J. Pickles, "Application of Urdhva Sutra”, Spiritual Study Group, Roorkee (India), 1984.

[3] Mrs. M. Ramalatha, Prof. D. Sridharan, "VLSI Based High Speed Karatsuba Multiplier for Cryptographic Applications Using Vedic Mathematics", 2007, IJSCI

[4] Thapliyal H. and Srinivas M.B., "High Speed Efficient N x N Bit Parallel Hierarchical Overlay Multiplier Architecture Based on Ancient Indian Vedic Mathematics", 2004, Vol.2, Transactions on Engineering, Computing and Technology,

[5] Jagadguru Swami Sri Bharati Krsna Tirthatji Maharaja, "Vedic Mathematics: sixteen simple mathematical formulae from the Veda", pp. 5-45, Motilal Banarsidas Publishers, Delhi, 2009.

[6] Anshul Khare, Dr. V.N. Yadav, Vandana Shikarwar, “ALU Using Area Optimized Vedic Multiplier", July 2014, pp. 207-210, IJERA.

[7] S.G. Dani, "Vedic Maths facts and myths", Vol 4/6, January 2001, pp. 20-21, One India One People.

[8] Himanshu Thapliyal, "Vedic Mathematics for Faster Mental Calculations and High Speed VLSI Arithmetic", Invited talk at IEEE Computer Society Student Chapter, Nov 14 2008, University of South Florida, Tampa, FL,

[9] Honey Durga Tiwari, Ganzorig Gankhuyag, Chan Mo Kim, Yong Beom Cho, "Multiplier Design based on Ancient Indian Vedic Mathematics",Nov. 2008, pp.6568, International SoC Design Conference,

[10] PUCKNULL. D.A., and ESIIRAGHIAN, K. "Basic VLSI design”, Prentice Hall, New Jersey, USA, 1994. 
[11] Devika, K. Sethi and R.Panda, "Vedic Mathematics Based Multiply Accumulate Unit," International Conference on Computational Intelligence and Communication Systems, pp.754-757, Nov. 2011CICN 2011.

[12] Purushottam D. Chidgupkar and Mangesh T. Karad, "The Implementation of Vedic Algorithms in Digital Signal Processing", Global J. of Engng. Educ., Vol.8, No.2, 2004, UICEE Published in Australia.
[13] Charles E. Stroud, "A Designer"s Guide to Built-In SelfTest", University of North Carolina at Charlotte, 2002, Kluwer Academic Publishers New York, Boston, Dordrecht, London, Moscow.

[14] www.xilinx.com 